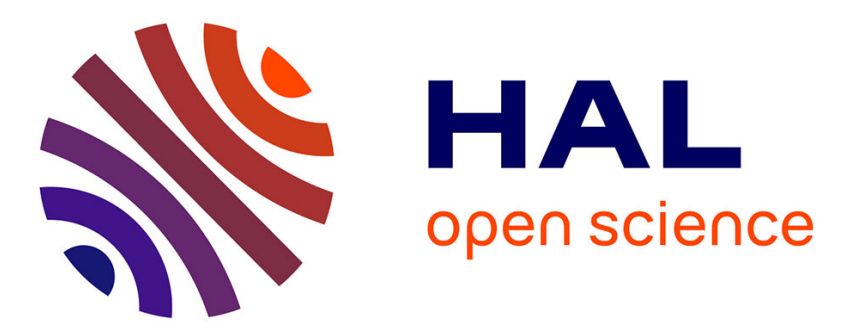

\title{
Analyse de la fonction de transfert générale d'un amplificateur à choppers
}

\author{
Hervé Aubert, M. Bazilinski
}

\section{To cite this version:}

Hervé Aubert, M. Bazilinski. Analyse de la fonction de transfert générale d'un amplificateur à choppers. Revue de Physique Appliquée, 1967, 2 (4), pp.301-308. 10.1051/rphysap:0196700204030100 . jpa-00242809

\section{HAL Id: jpa-00242809 https://hal.science/jpa-00242809}

Submitted on 1 Jan 1967

HAL is a multi-disciplinary open access archive for the deposit and dissemination of scientific research documents, whether they are published or not. The documents may come from teaching and research institutions in France or abroad, or from public or private research centers.
L'archive ouverte pluridisciplinaire HAL, est destinée au dépôt et à la diffusion de documents scientifiques de niveau recherche, publiés ou non, émanant des établissements d'enseignement et de recherche français ou étrangers, des laboratoires publics ou privés. 


\title{
ANALYSE DE LA FONGTION DE TRANSFERT GÉNÉRALE D'UN AMPLIFIGATEUR A GHOPPERS
}

\author{
Par H. AUBERT et M. BAZILINSKI.
}

\begin{abstract}
Résumé. - La fonction de transfert d'un ensemble d'amplification à choppers présente, dans la gamme d'utilisation, une déformation inhérente au principe de cet appareil, même si l'amplificateur proprement dit est supposé parfait, phénomène dont il semble que la théorie n'avait pas été élaborée.

Par un passage à la limite laissant valables les conclusions pratiques, on montre que, bien que les processus mis en jeu soient essentiellement non linéaires, cette fonction obéit au principe de superposition. On la met sous la forme d'un produit dont les trois facteurs représentent respectivement le gain à fréquence nulle, le gain relatif complexe filtre non compris et l'effet du filtre.

On en déduit des formules permettant de choisir au mieux les caractéristiques des composants en vue de minimiser ce défaut particulièrement gênant dans les travaux de recherche exigeant une réponse transitoire aussi fidèle que possible.

On montre en outre que l'imperfection de l'amplificateur proprement dit entraîne un décalage entre l'abscisse du minimum d'amplitude et celle du passage à zéro de la phase.

Les formules obtenues se simplifient beaucoup dans nombre de cas pratiques en donnant des approximations en général suffisantes.

Abstract. - The transfer function of a chopper-modulated amplifier is derived by the authors. The expressions obtained show the possible significant variations of amplitude and phase within the usable frequency range even if the amplifying component is practically perfect.

The mathematical analysis of this behavior does not appear to have been made in detail at the present time.

It is shown that despite the nonlinearity of the current versus time relationship, the principle of superposition may be applied to the transfer function, which can be written in the form of a product of three factors. These factors represent respectively : the gain at zero frequency, the complex gain without filter, and the effect of the filter.

Formulae for optimization of values of different components are given for improvement of transient response. It is shown, moreover, how the imperfections of the amplifying component affect the characteristics of amplitude and phase.

The formulae obtained can be simplified in most practical cases, generally giving satisfactory approximations.
\end{abstract}

Généralités. - UTILITÉ DE L'ÉTUDE. - Malgré les progrès technologiques récents concernant la dérive des amplificateurs continus proprement dits, l'usage des choppers, mécaniques, optiques ou par transistors à effet de champ par exemple, est encore nécessaire dans bien des cas.

Or, un raisonnement hâtif assimilant la modulation et la démodulation par choppers à de simples déplacements du spectre, tels qu'on les pratique en téléphonie à courants porteurs, ne rend pas compte d'un phénomène gênant, propre au fonctionnement des choppers.

En effet, même en utilisant entre les choppers un amplificateur parfait, la fonction de transfert obtenue présente, bien en deçà de la fréquence de découpage, dans une région utile si l'on s'intéresse à la réponse transitoire, une baisse systématique du module suivie d'une remontée, accompagnées d'un déphasage assez rapidement variable, s'annulant avec la dérivée du module. Et si l'amplificateur est imparfait cette coïncidence n'est même plus assurée.

Cet accident, qui peut limiter ou compliquer l'usage des choppers dans certaines applications, est lié aux valeurs des constantes de temps associées aux choppers, et au rapport des temps d'ouverture et de fermeture, rapport dont dépend le gain à fréquence nulle.

Il a semblé utile d'étudier ce phénomène en détail pour établir des relations dont l'emploi permettrait à l'ingénieur d'étude de minimiser ce défaut et d'optimiser son projet.

Accessoirement, cette étude montre que, bien que les processus mis en jeu soient essentiellement non linéaires, la fonction de transfert globale obéit au principe de superposition : l'ensemble du système se comporte, dans la bande utile, comme un système linéaire. 


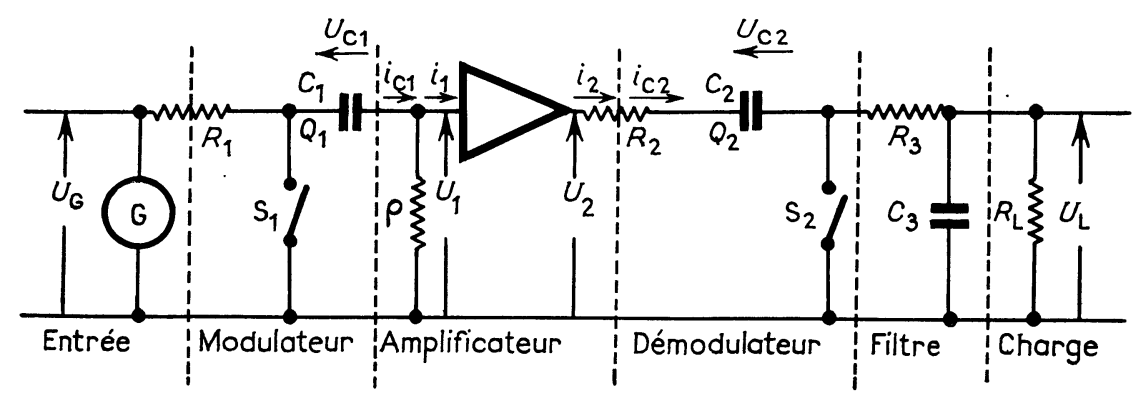

Frg. 1. - Schéma de principe de l'amplificateur à choppers.

PRINGIPE DE LA MÉTHODE. - L'analyse rigoureuse, par raccordements successifs des conditions finales et initiales de deux équations distinctes, est pratiquement inextricable. Mais il est possible, parce que la fréquence de découpage peut être choisie très au-delà de la limite supérieure du spectre à amplifier, de ramener le problème à des calculs simples grâce aux artifices suivants :

On définit, d'une façon rigoureuse par un passage à la limite, des composantes lentes et des composantes rapides, notions qui prolongent celles de composante continue et de composante alternative dans le cas du régime établi. Ces composantes lentes, ainsi que les amplitudes et les enveloppes des composantes rapides, sont alors traitées légitimement comme des fonctions continues dont les transformées de Laplace ont une expression simple. La fréquence de découpage, que l'on a fait tendre vers l'infini, ne figure plus dans les calculs. On la réintroduit seulement à la fin pour le calcul de l'ondulation résiduelle du signal de sortie.

La figure 1 donne la décomposition du dispositif en divers éléments ainsi que les conventions de signe adoptées.

La figure 2 donne, dans le cas particulier d'une tension d'entrée en échelon, l'allure des tensions obtenues aux bornes de $\mathrm{C}_{1}$ et de $\rho$, et de leurs composantes. L'examen de ces courbes facilite la compréhension des définitions données plus loin.

Notations ET DÉfinitions :

$T_{\mathrm{o}}=1 / f_{\mathrm{o}}=$ période de découpage,

$x T_{0} \quad=$ durée de fermeture des choppers,

$(1-x) T_{\mathrm{o}}=$ durée d'ouverture des choppers,

$u_{\mathrm{G}} \quad=$ tension à amplifier,

$u_{\mathrm{C} 1} \quad=$ tension aux bornes de $\mathrm{C}_{1}$,

$Q_{1}=$ charge de $\mathrm{G}_{1}$ à l'instant $t$,

$\Delta^{\prime} Q_{1} \quad=$ variation de $Q_{1}$ pendant la durée $(1-x) T_{0}$ de la charge,

$\Delta^{\prime \prime} Q_{1} \quad=$ variation de $Q_{1}$ pendant la durée $x T_{0}$ de la décharge,

$u_{1}=$ tension discontinue appliquée à l'entrée de l'amplificateur,

$\nu \quad=$ limite supérieure du spectre à amplifier.
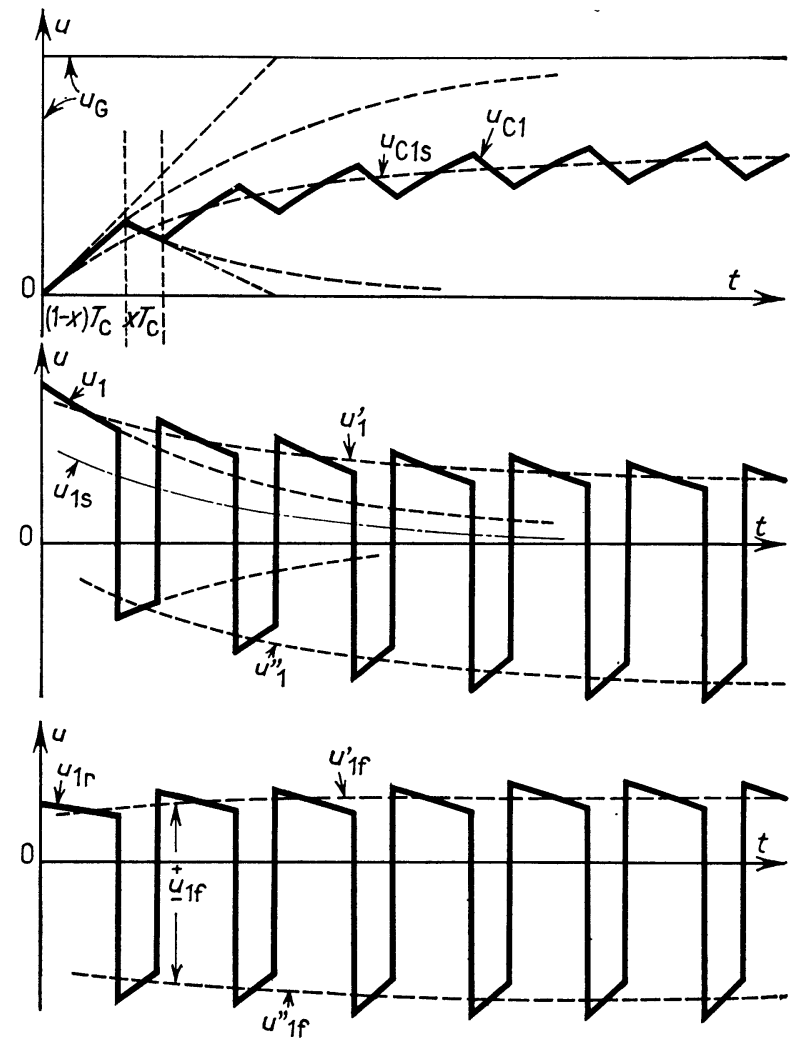

Frg. 2. - Décomposition des signaux en composantes lentes et rapides. Exemple pour $x=1 / 3, C_{1}\left(R_{1}+\rho\right)=$ $2 T_{0}, \rho / R_{1}=2, \quad u_{\mathrm{G}}=$ échelon unité.

$f_{0}$ étant bien supérieure à $\nu$, le spectre total se compose de deux parties nettement séparées, couvrant respectivement :

1) le spectre utile allant de 0 à $v$;

2) l'ensemble des domaines $n f_{0} \pm \nu$, où $n f_{0}$ désigne le $n^{\text {ieme }}$ harmonique de $f_{0}$.

On peut donc considérer chaque fonction du temps comme la somme d'une fonction à variation lente dont le spectre s'étend de 0 à $\nu$ et d'une fonction à 
variation rapide possédant le reste du spectre, ce qui permet de poser :

$u_{18}=$ fonction continue interpolant les points d'abscisse $(n+1 / 2) T_{\mathrm{c}}$ et d'ordonnée $\frac{1}{T_{\mathrm{c}}} \int_{n T_{\mathrm{c}}}^{(n+1) T_{\mathrm{o}}} u_{1} \mathrm{~d} t$ (suite des centres de gravité de l'ensemble des deux arcs de courbe de chacune des périodes successives complètes) que nous appellerons composante lente de $u_{1}$ (se reporter à la figure 2),

$u_{1 \mathrm{r}}=u_{1}-u_{1 \mathrm{~s}}$ que nous appellerons composante rapide de $u_{1}$,

$u_{1}^{\prime}=$ fonction continue interpolant les points d'abscisse $\left(n+\frac{1-x}{2}\right) T_{\mathrm{c}}$ et d'ordonnée

$$
\frac{1}{(1-x) T_{\mathrm{c}}} \int_{n T_{\mathrm{c}}}^{(n+1-x) T_{\mathrm{c}}} u_{1} \mathrm{~d} t
$$

(suite des centres de gravité des arcs de courbe supérieurs seulement de chacune des périodes successives) que nous appellerons enveloppe supérieure de $u_{1}$,

$u_{1}^{\prime \prime}=$ fonction continue interpolant les points d'abscisse $\left(n+1-\frac{x}{2}\right) T_{\mathrm{c}}$ et d'ordonnée

$$
\frac{1}{x T_{\mathrm{c}}} \int_{(n+1-x) T_{\mathrm{c}}}^{(n+1) T_{\mathrm{e}}} u_{1} \mathrm{~d} t
$$

(suite des centres de gravité des arcs de courbe inférieurs seulement de chacune des périodes successives) que nous appellerons enveloppe inférieure de $u_{1}$,

$u_{1 \mathrm{f}}^{\prime}=$ fonction définie à partir de $u_{1 \mathrm{r}}$ de la même façon que $u_{1}^{\prime}$ est définie à partir de $u_{1}$. Nous l'appellerons enveloppe supérieure de la composante rapide,

$u_{1 \mathrm{f}}^{\prime \prime}=$ fonction définie à partir de $u_{1 \mathrm{r}}$ de la même façon que $u_{1}^{\prime \prime}$ est définie à partir de $u_{1}$. Nous l'appellerons enveloppe inférieure de la composante rapide,

$u_{1 \mathrm{f}}=u_{1 \mathrm{f}}^{\prime}-u_{1 \mathrm{f}}^{\prime \prime}$, fonction continue que nous appellerons amplitude de crête à crête de la composante rapide.

La façon dont sont faites ces interpolations n'est pas exactement précisée car elle n'interviendra pas dans la suite. On peut par exemple supposer qu'elle correspond au passage à la limite pour $m \rightarrow \infty$ de l'interpolation entre $m$ points consécutifs au moyen d'un polynôme de degré $m-1$.

Les mêmes notations seront reprises pour les tensions sortant de l'amplificateur, avec l'indice 2 au lieu de l'indice 1 .

Etude du circuit d'entrée. - Approximation. Faisons tendre $f_{\mathrm{o}}$ vers l'infini. Les définitions données ci-dessus deviennent indépendantes du mode d'interpolation choisi. Ceci suppose que l'on utilisera des constantes de temps associées aux choppers très grandes devant $T_{0}$. L'expérience vérifie alors les résultats de la théorie avec une bonne approximation : $u_{1 \mathrm{~s}}=\underset{\text { continue }),}{\text { limite pour }} T_{\mathrm{c}} \rightarrow 0 \mathrm{de} \frac{1}{T_{\mathrm{c}}} \int_{t}^{t+T_{\mathrm{o}}} u_{1} \mathrm{~d} t \quad\left(u_{1}\right.$ dis$u_{1 \mathrm{r}}=u_{1}-u_{1 \mathrm{~s}}$ est une tension en créneaux dont la valeur moyenne sur $T_{\mathrm{c}}$ est nulle.

Nous conservons les mêmes notations pour désigner après passage à la limite les fonctions

$$
u_{1}^{\prime}, \ldots, u_{1 \mathrm{f}}^{\prime \prime} \text { et } u_{1 \mathrm{f}} \text {. }
$$

Les transformées de Laplace correspondantes seront désignées par les mêmes symboles mais avec des $U$ majuscules.

Marghe dU Galgul :

$$
u_{1 \mathrm{~s}}=\frac{1}{T_{\mathrm{c}}}\left[\int_{n T_{\mathrm{e}}}^{(n+1-x) T_{\mathrm{e}}} u_{1} \mathrm{~d} t+\int_{(n+1-x) T_{\mathrm{e}}}^{(n+1) T_{\mathrm{c}}} u_{1} \mathrm{~d} t\right]
$$

mais $u_{1}=u_{1}^{\prime}$ dans la première somme, tandis que $u_{1}=u_{1}^{\prime \prime}$ dans la deuxième, et comme ces fonctions sont continues elles sortent du signe somme pour $T_{\mathrm{c}} \rightarrow 0$, ce qui donne :

$$
u_{1 \mathrm{~s}}=u_{1}^{\prime}(1-x)+u_{1}^{\prime \prime} x .
$$

Le même calcul fait sur $u_{1 \mathrm{r}}$ donne :

$$
u_{1 \mathrm{f}}^{\prime}(1-x)+u_{1 \mathrm{f}}^{\prime \prime} x=0 \text {. }
$$

On obtient aussi simplement les relations :

$$
u_{1}^{\prime}-u_{1 \mathrm{f}}^{\prime}=u_{1 \mathrm{~s}} ; \quad u_{1}^{\prime \prime}-u_{1 \mathrm{f}}^{\prime \prime}=u_{1 \mathrm{~s}} ; \quad u_{1 \mathrm{f}}=u_{1}^{\prime}-u_{1}^{\prime \prime}
$$

fonctions que nous exprimerons à partir de la tension $u_{\mathrm{C} 1}$.

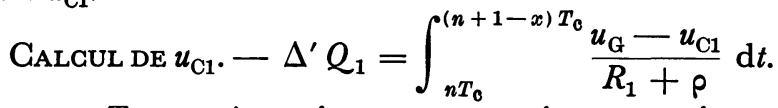

Comme $T_{\mathrm{c}}$ est très petit, $u_{\mathrm{G}}$ et $u_{\mathrm{C} 1}$ qui sont continues sortent du signe somme :

$$
\Delta^{\prime} Q_{1}=\frac{u_{\mathrm{G}}-u_{\mathrm{C} 1}}{R_{1}+\rho}(1-x) T_{\mathrm{c}} .
$$

On a de même :

$$
\Delta^{\prime \prime} Q_{1}=-\frac{u_{\mathrm{C} 1}}{\rho} x T_{\mathrm{c}}
$$

soit sur une période complète :

$\Delta Q_{1}=\Delta^{\prime} Q_{1}+\Delta^{\prime \prime} Q_{1}$

$$
\begin{aligned}
& =\frac{u_{\mathrm{G}}-u_{\mathrm{C} 1}}{R_{1}+\rho}(1-x) T_{\mathrm{c}}-\frac{u_{\mathrm{C} 1}}{\rho} \times T_{\mathrm{c}} \\
& =u_{\mathrm{G}} \frac{1-x}{R_{1}+\rho} T_{\mathrm{c}}-u_{\mathrm{C} 1} \frac{\rho+x R_{1}}{\rho\left(R_{1}+\rho\right)} T_{\mathrm{c}} .
\end{aligned}
$$

Par ailleurs, on a $\Delta Q_{1}=C_{1} \Delta u_{\mathrm{C} 1}$, d'où l'équation :

$$
\frac{\Delta u_{\mathrm{C} 1}}{T_{\mathrm{c}}}+\frac{\rho+R_{1} x}{C_{1} \rho\left(R_{1}+\rho\right)} u_{\mathrm{C} 1}=\frac{1-x}{C_{1}\left(R_{1}+\rho\right)} u_{\mathrm{G}} .
$$

La dérivée de $u_{\mathrm{C} 1}(t)$ n'étant pas une fonction continue, appelons $u_{\mathrm{C} 10}(t)$ la valeur de $u_{\mathrm{C} 1}(t)$ à la fin de chaque période complète. Cette fonction n'est pas 
définie pour $t \neq n T_{\mathrm{c}}$, mais si $T_{\mathrm{c}} \rightarrow 0, u_{\mathrm{C} 1 \mathrm{o}}$ tend vers une fonction continue, et en prenant $T_{\mathrm{c}}$ comme infiniment principal $\mathrm{d} t$, il vient :

$$
\frac{\mathrm{d}}{\mathrm{d} t} u_{\mathrm{C} 1 \mathrm{o}}+\frac{\rho+R_{1} x}{C_{1} \rho\left(R_{1}+\rho\right)} u_{\mathrm{C} 1 \mathrm{o}}=\frac{1-x}{C_{1}\left(R_{1}+\rho\right)} u_{\mathrm{G}}
$$

(qu'il n'est pas question d'intégrer puisque $u_{\mathrm{G}}(t)$ n'est pas précisée).

Posons la constante de temps $\frac{C_{1} \rho\left(R_{1}+\rho\right)}{\rho+R_{1} x}=\tau_{\mathrm{M} 2}$ et désignons par des capitales les transformées de Laplace : $A(s)=\int_{0}^{\infty} a(t) \mathrm{e}^{-s t} \mathrm{~d} t$. En supposant $Q_{1}(\mathrm{o})=0$, il vient :

$$
U_{\mathrm{C} 1 \mathrm{o}}=\frac{1-x}{C_{1}\left(R_{1}+\rho\right)} \frac{\tau_{\mathrm{M} 2}}{1+s \tau_{\mathrm{M} 2}} U_{\mathrm{G}}
$$

Si les dérivées $\frac{\mathrm{d}}{\mathrm{d} t} u_{\mathrm{C} 1}$ et $\frac{\mathrm{d}}{\mathrm{d} t} u_{\mathrm{C} 10}$ sont différentes, les fonctions elles-mêmes ne diffèrent en tout point au maximum que d'un infiniment petit de l'ordre de $\frac{u_{\mathrm{C} 1} x}{C_{1}} \mathrm{~d} t\left(\right.$ limite de $\left.\frac{\Delta^{\prime \prime} Q_{1}}{C_{1}}\right)$ et l'on peut remplacer $u_{\mathrm{C} 1}$ par $u_{\text {Clo }}$ partout où sa dérivée n'intervient pas. Le schéma montre alors que : $u_{1}=\left(u_{\mathrm{G}}-u_{\mathrm{C} 1 \mathrm{o}}\right) \frac{\rho}{R_{1}+\rho}$ quand $\mathrm{S}_{1}$ est ouvert, $u_{1}=-u_{\mathrm{Clo}}$ quand il est fermé. Ces valeurs de $u_{1}$ ne sont autres, à $\mathrm{d} u_{1}$ près, que $u_{1}^{\prime}$ et $u_{1}^{\prime \prime}$ respectivement. On peut en tirer :

$u_{1 \mathrm{~s}}=u_{1}^{\prime}(1-x)+u_{1}^{\prime \prime} x=\frac{(1-x) \rho}{R_{1}+\rho} u_{\mathrm{G}}-\frac{R_{1} x+\rho}{R_{1}+\rho} u_{\mathrm{C} 1}$

$u_{1 \mathrm{f}}=u_{1}^{\prime}-u_{1}^{\prime \prime}=\frac{\rho}{R_{1}+\rho} u_{\mathrm{G}}+\frac{R_{1}}{R_{1}+\rho} u_{\mathrm{C} 1}$

$u_{1 \mathrm{f}}^{\prime}=u_{1}^{\prime}-u_{1 \mathrm{~s}}=\frac{\rho x}{R_{1}+\rho} u_{\mathrm{G}}+\frac{R_{1} x}{R_{1}+\rho} u_{\mathrm{C} 1}$

$u_{1 \mathrm{f}}^{\prime \prime}=u_{1}^{\prime \prime}-u_{1 \mathrm{~s}}=-\frac{(1-x) \rho}{R_{1}+\rho} u_{\mathrm{G}}+\frac{R_{1} x-R_{1}}{R_{1}+\rho} u_{\mathrm{C} 1}$.

En posant $\tau_{\mathrm{M} 1}=C_{1} \rho$ et en utilisant la définition de $\tau_{\mathrm{M} 2}$, les transformées correspondantes s'écrivent facilement :

$$
\begin{aligned}
U_{1 \mathrm{~s}} & =\frac{\rho(1-x)}{R_{1}+\rho} \frac{s \tau_{\mathrm{M} 2}}{1+\tau s_{\mathrm{M} 2}} \mathrm{U}_{\mathrm{G}} \\
U_{1 \mathrm{f}} & =\frac{\rho}{R_{1} x+\rho} \frac{1+s \tau_{\mathrm{M} 1}}{1+s \tau_{\mathrm{M} 2}} \mathrm{U}_{\mathrm{G}} \\
U_{1 \mathrm{f}}^{\prime} & =\frac{\rho x}{R_{1} x+\rho} \frac{1+s \tau_{\mathrm{M} 1}}{1+s \tau_{\mathrm{M} 2}} \mathrm{U}_{\mathrm{G}} \\
U_{1 \mathrm{f}}^{\prime \prime} & =-\frac{\rho(1-x)}{R_{1} x+\rho} \frac{1+s \tau_{\mathrm{M} 1}}{1+s \tau_{\mathrm{M} 2}} \mathrm{U}_{\mathrm{G}} .
\end{aligned}
$$

Il était nécessaire de calculer toutes ces expressions, car l'amplificateur n'a pas le même gain pour la composante lente $u_{1 \mathrm{~s}}$ que pour la composante rapide $u_{1 \mathrm{r}}$ et parce que les deux enveloppes de $u_{1 \mathrm{r}}$ interviennent séparément dans le fonctionnement du démodulateur.

Etude du circuit de sortie. - Amplification. On voit que $U_{1 \mathrm{~s}}$ est nul à l'origine et limité à l'intervalle $0<s<2 \pi j v$. Le spectre de $u_{1 \mathrm{~s}}$ tombe donc dans la région où l'amplificateur alternatif présente un gain faible croissant et complexe, et la composante lente $u_{1 \mathrm{~s}}$ sera distordue et peu amplifiée.

Par contre, le spectre de $u_{1 \mathrm{r}}$ s'étend de $f_{\mathrm{c}}-\nu$ à $n f_{\mathrm{c}}+v$, le rang $n$ de l'harmonique $n f_{\mathrm{c}}$ étant élevé car les capacités shunt parasites (non figurées sur le schéma) forment avec $R_{1}$ et $\rho$ des constantes de temps faibles devant $T_{\mathrm{c}}$.

On donnera donc à l'amplificateur une fréquence de coupure supérieure assez grande pour éviter d'arrondir sensiblement les angles de $u_{1}$ après amplification et nous raisonnerons comme si $u_{1 \mathrm{r}}$ était amplifiée sans aucune distorsion, ce que l'on sait faire pratiquement.

Pour préciser, l'expression du gain de l'amplificateur étant de la forme :

$$
K_{\mathrm{A}} G_{\mathrm{A}}(s)=K_{\mathrm{A}} \frac{s^{m}+a_{m-1} s^{m-1}+\ldots a_{1} s+a_{0}}{s^{n}+b_{n-1} s^{n-1}+\ldots b_{1} s+b_{0}}
$$

on raisonne comme si on avait : $m=n, a_{0}=0, b_{0} \neq 0$ et les autres coefficients tels que $G_{\mathbf{A}}(s)$ soit pratiquement égal à 1 dans toute la gamme utile pour respecter la forme de $u_{1 \mathrm{r}}$.

Notons que la contre-réaction utilisée pour stabiliser thermiquement les étages à transistors fait décroître le gain aux fréquences basses. Quand elle est appliquée à l'étage d'entrée, elle rend en outre complexe l'impédance d'entrée aux basses fréquences. Cet effet n'est pas pris en considération dans les présents calculs.

Signal SORTANT DE L'AMPLIFIGATEUR. - La tension

$$
u_{2}(t)=K_{\mathrm{A}} G_{\mathrm{A}}\left(\frac{\mathrm{d}}{\mathrm{d} t}\right) u_{1 \mathrm{~s}}(t)+K_{\mathrm{A}} u_{1 \mathrm{r}}(t)
$$

a pour enveloppes :

$$
u_{2}^{\prime}(t)=K_{\mathrm{A}} G_{\mathrm{A}}\left(\frac{\mathrm{d}}{\mathrm{d} t}\right) u_{1 \mathrm{~s}}(t)+K_{\mathrm{A}} u_{1 \mathrm{f}}^{\prime}(t)
$$

et :

$$
u^{\prime \prime}(t)=K_{\mathrm{A}} G_{\mathrm{A}}\left(\frac{\mathrm{d}}{\mathrm{d} t}\right) u_{1 \mathrm{~s}}(t)+K_{\mathrm{A}} u_{1 \mathrm{f}}^{\prime \prime}(t)
$$
(dans ces trois équations, $G_{\mathrm{A}}\left(\frac{\mathrm{d}}{\mathrm{d} t}\right)$ est un opérateur
portant sur $u$ ).

Prenons les transformées de ces équations et portons-y les valeurs obtenues plus haut pour $U_{1 \mathrm{~s}}, U_{1 \mathrm{f}}^{\prime}$ et $U_{1 \mathrm{f}}^{\prime \prime}$, il vient, tous calculs faits :

$$
\begin{aligned}
U_{2}^{\prime}(s)=K_{\mathrm{A}}\{ & \frac{(1-x) \rho}{R_{1}+\rho} s \tau_{\mathrm{M} 2} G_{\mathrm{A}}(s) \\
& \left.\quad+\frac{x \rho}{\rho+x R_{1}}\left(1+s \tau_{\mathrm{M} 1}\right)\right\} \frac{1}{1+s \tau_{\mathrm{M} 2}} U_{\mathrm{G}}(s)
\end{aligned}
$$


et :

$$
\begin{aligned}
U_{2}^{\prime \prime}(s)=K_{\mathrm{A}} & \left\{\frac{(1-x) \rho}{R_{1}+\rho} s \tau_{\mathrm{M} 2} G_{\mathrm{A}}(s)\right. \\
& \left.\quad-\frac{(1-x) \rho}{\rho+x R_{1}}\left(1+s \tau_{\mathrm{M} 1}\right)\right\} \frac{1}{1+s \tau_{\mathrm{M} 2}} U_{\mathrm{G}}(s) .
\end{aligned}
$$

Démodulation. - En appliquant la même méthode que pour le circuit d'entrée, il vient :

$$
\begin{aligned}
& s U_{\mathrm{C} 2}=\frac{U_{2}^{\prime}-U_{\mathrm{C} 2}-U_{\mathrm{L}}}{C_{2}\left(R_{2}+R_{3}\right)}(1-x)+\frac{U_{2}^{\prime \prime}-U_{\mathrm{C} 2}}{C_{2} R_{2}} x \\
& s U_{\mathrm{L}}=\frac{U_{2}^{\prime}-U_{\mathrm{C} 2}-U_{\mathrm{L}}}{C_{3}\left(R_{2}+R_{3}\right)}(1-x)-U_{\mathrm{L}} \frac{R_{3}+x R_{\mathrm{L}}}{C_{3} R_{3} R_{\mathrm{L}}} .
\end{aligned}
$$

Éliminons $U_{\mathrm{C} 2}$ entre ces deux équations. Il vient, tous calculs faits :

$$
U_{\mathrm{L}}=\frac{x(1-x) R_{3} R_{\mathrm{L}}\left[\left(1+s \tau_{\mathrm{D} 1}\right) U_{2}^{\prime}-U_{2}^{\prime \prime}\right]}{R_{3}\left(R_{2}+x R_{3}\right)+x R_{\mathrm{L}}\left(R_{2}+R_{3}\right)} \frac{1}{1+s \tau_{\mathrm{D} 2}} \frac{1}{1+s \tau_{\mathrm{F}}}
$$

où $\tau_{\mathrm{D} 1}, \tau_{\mathrm{D} 2}$ et $\tau_{\mathrm{F}}$ sont des constantes de temps dont les expressions sont données plus loin. Portons dans $U_{\mathrm{L}}$ les valeurs obtenues plus haut pour $U_{2}^{\prime}$ et $U_{2}^{\prime \prime}$. Il vient, tous calculs faits :

$$
U_{\mathrm{L}}=\frac{(1-x) \rho}{\rho+x R_{1}} \frac{K_{\mathrm{A}}}{1+\frac{R_{2}}{R_{3}}+\frac{R_{2}+x R_{3}}{x R_{\mathrm{L}}}} \frac{(1-x) \tau_{\mathrm{M} 1} \tau_{\mathrm{D} 1} s^{2} G_{\mathrm{A}}(s)+x \tau_{\mathrm{M} 1} \tau_{\mathrm{D} 1} s^{2}+\left(\tau_{\mathrm{M} 1}+x \tau_{\mathrm{D} 1}\right) s+1}{\left(1+s \tau_{\mathrm{M} 2}\right)\left(1+s \tau_{\mathrm{D} 2}\right)\left(1+2 \tau_{\mathrm{F}}\right)} \mathrm{U}_{\mathrm{G}}
$$

avec :

$$
\begin{gathered}
\tau_{\mathrm{M} 1}=C_{1} \rho ; \quad \tau_{\mathrm{M} 2}=C_{1} \rho \frac{\rho+R_{1}}{\rho+x R_{1}} ; \quad \tau_{\mathrm{D} 1}=\frac{C_{2} R_{2}}{x} \\
\tau_{\mathrm{D} 2}=\frac{C_{\mathrm{F}} R_{\mathrm{F}}}{2}(1-\sqrt{1-\varepsilon}) \\
\tau_{\mathrm{F}}=\frac{C_{\mathrm{F}} R_{\mathrm{F}}}{2}(1+\sqrt{1-\varepsilon})
\end{gathered}
$$

où l'on a posé :

$$
C_{\mathrm{F}}=C_{3}\left[1+\frac{C_{2}}{C_{3}} \frac{R_{2}}{x R_{\mathrm{L}}} \frac{R_{3}\left(R_{2}+R_{3}\right)+R_{\mathrm{L}}\left(R_{3}+x R_{\mathrm{L}}\right)}{R_{3}\left(R_{2}+x R_{3}\right)+R_{\mathrm{L}}\left(R_{2}+R_{3}\right)}\right]
$$$$
R_{\mathrm{F}}=R_{3} \frac{R_{\mathrm{L}}\left(R_{2}+x R_{3}\right)}{R_{3}\left(R_{2}+x R_{3}\right)+x R_{\mathrm{L}}\left(R_{2}+R_{3}\right)}
$$

et :

$$
\varepsilon=4 \frac{C_{2}}{C_{\mathrm{F}}} \frac{C_{3}}{C_{\mathrm{F}}} \frac{R_{2}}{R_{3}} \frac{R_{2}+R_{3}}{R_{2}+x R_{3}}\left[\frac{R_{3}}{R_{\mathrm{L}}}+\frac{x\left(R_{2}+R_{3}\right)}{R_{2}+x R_{3}}\right] .
$$

Le taux d'ondulation résiduelle, valeur de crête à crête, rapporté à la tension utile de sortie, est donné par :

$$
\eta=\frac{x}{C_{3} f_{\mathrm{c}}} \frac{R_{3}+R_{\mathrm{L}}}{R_{3} R_{\mathrm{L}}} .
$$

On voit que la fonction de transfert de l'ensemble apparaît comme le produit de trois facteurs assez bien séparés :

$$
\frac{U_{\mathrm{L}}(s)}{U_{\mathrm{G}}(s)}=K_{\mathrm{T}} \cdot G_{\mathrm{p}}(s) \cdot G_{\mathrm{F}}(s)
$$

$$
\text { où : } \begin{aligned}
K_{\mathrm{T}}=\frac{(1-x) \rho}{\rho+x R_{1}} \frac{K_{\mathrm{A}}}{1+\frac{R_{2}}{R_{3}}+\frac{R_{2}+x R_{3}}{x R_{\mathrm{L}}}} \text { représente le gain en continu } \\
G_{\mathrm{p}}(s)=\frac{(1-x) \tau_{\mathrm{M} 1} \tau_{\mathrm{D} 1} s^{2} G_{\mathrm{A}}(s)+x \tau_{\mathrm{M} 1} \tau_{\mathrm{D} 1} s^{2}+\left(\tau_{\mathrm{M} 1}+x \tau_{\mathrm{D} 1}\right) s+1}{\left(1+s \tau_{\mathrm{M} 2}\right)\left(1+s \tau_{\mathrm{D} 2}\right)}
\end{aligned}
$$

le gain relatif complexe en régime variable, effet du filtre non compris (où $\tau_{\mathrm{M} 2}$ dépend de $C_{3}$ et $R_{3}$ contenus dans le filtre), et : $G_{\mathrm{F}}(s)=\frac{1}{1+s \tau_{\mathrm{F}}}$ le gain relatif complexe du filtre en régime variable (où $\tau_{\mathrm{F}}$ dépend de $R_{2}$ et $C_{2}$ qui ne sont pas dans le filtre).

Présentation des résultats. - Approximations simPLIFICATRICES. - 1) Si on ne prend pas en compte la baisse de gain aux basses de l'amplificateur (action sur la composante lente), on peut écrire : $G_{\mathbf{A}}(j \omega)=1$ quel que soit $\omega$. En posant alors :

$$
\begin{gathered}
\tau_{1}=\sqrt{\tau_{\mathrm{M} 1} \tau_{\mathrm{D} 1}} \text { et } \lambda_{1}=1 / 2\left(\sqrt{\frac{\tau_{\mathrm{M} 1}}{\tau_{\mathrm{D} 1}}}+x \sqrt{\frac{\tau_{\mathrm{D} 1}}{\tau_{\mathrm{M} 1}}}\right) \\
\text { REVUE DE PHYSIQUE APPLQUÉE. }- \text { T. 2. } \mathrm{N}^{\mathrm{O}} \text { 4. DÉCEMBRE } 1967 .
\end{gathered}
$$

il vient :

$$
\left|G_{\mathrm{p}}\right|=\sqrt{\frac{\left(1-\tau_{1}^{2} \omega^{2}\right)^{2}+4 \omega^{2} \lambda_{1}^{2} \tau_{1}^{2}}{\left(1+\tau_{\mathrm{M} 2}^{2} \omega^{2}\right)\left(1+\tau_{\mathrm{D} 2}^{2} \omega^{2}\right)}}
$$

et $\varphi(\omega)=\operatorname{Arctg} \frac{2 \lambda_{1} \omega \tau_{1}}{1-\omega^{2} \tau_{1}^{2}}-\operatorname{Arctg} \frac{\left(\tau_{M 2}+\tau_{D 2}\right) \omega}{1-\omega^{2} \tau_{M 2} \tau_{D}}$ expressions sur lesquelles nous reviendrons. 
2) Dans de nombreuses applications, les valeurs des composants sont telles que les expressions obtenues peuvent se simplifier considérablement. Généralement, $\varepsilon$ est $<1 / 2$, ce qui permet de calculer $\tau_{\mathrm{F}}$ et $\tau_{\mathrm{D} 2}$ avec une approximation de 10 à $15 \%$ au moyen des expressions suivantes :

$\tau_{\mathrm{F}} \cong C_{\mathrm{F}} R_{\mathrm{F}} \quad$ et $\quad \tau_{\mathrm{D} 2} \cong \frac{C_{\mathrm{F}} R_{\mathrm{F}}}{4} \varepsilon \cong C_{2} R_{2} \frac{R_{2}+R_{3}}{R_{2}+x R_{3}} \frac{C_{3}}{C_{\mathrm{F}}}$.

$\mathrm{Si}$, en outre, $C_{3} \cong C_{\mathrm{F}}$, on a :

$$
\begin{array}{cc} 
& \tau_{\mathrm{F}}=C_{3} R_{3} \frac{R_{\mathrm{L}}\left(R_{2}+x R_{3}\right)}{R_{3}\left(R_{2}+x R_{3}\right)+x R_{\mathrm{L}}\left(R_{2}+R_{3}\right)} \\
\text { et } \quad \tau_{\mathrm{D} 2}=C_{2} R_{2} \frac{R_{2}+R_{3}}{R_{2}+x R_{3}} .
\end{array}
$$

Si $R_{2}$ impédance de sortie de l'amplificateur est faible devant la charge, ce qui sera souvent le cas, ces formules peuvent encore se simplifier : pour $R_{2} / R_{3} \ll 1$ le gain continu devient :

$$
K_{\mathrm{T}}=\frac{\rho(1-x)}{\rho+x R_{1}} \frac{K_{\mathrm{A}}}{1+\frac{R_{3}}{R_{\mathrm{L}}}}
$$

ce qui montre que si $x$ décroît le gain $K_{\mathrm{T}}$ croît. De plus :

$$
\tau_{\mathrm{D} 2} \cong \tau_{\mathrm{D} 1}=\frac{C_{2} R_{2}}{x} \quad \text { et } \quad \tau_{\mathrm{F}} \cong C_{3} \frac{R_{3} R_{\mathrm{L}}}{R_{3}+R_{\mathrm{L}}} .
$$

Si les conditions $\varepsilon \ll 1 / 2$ et $C_{\mathrm{F}} \cong C_{3}$ sont satisfaites, il est possible de calculer directement la constante de temps $\tau_{F}$ du filtre à partir du taux d'ondulation résiduelle toléré $\eta$ :

$$
\tau_{\mathrm{F}}=\frac{x}{\eta f_{\mathrm{c}}} \frac{R_{3}\left(R_{2}+x R_{3}\right)+x R_{\mathrm{L}}\left(R_{2}+R_{3}\right)}{\left(R_{2}+x R_{3}\right)\left(R_{3}+R_{\mathrm{L}}\right)} .
$$

La figure 3 évoque la forme de l'ondulation résiduelle et rappelle la définition de $\eta=\frac{\Delta u_{\mathrm{L}}}{u_{\mathrm{L}}}$.

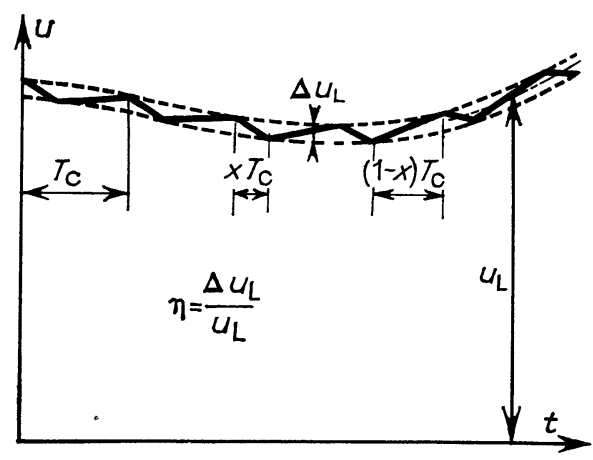

FIG. 3.

Forme de l'ondulation résiduelle à la sortie.

DisTORSION DE FRÉQuence ET DE PHASE. - Il est important de l'examiner, car la réponse transitoire intervient dans beaucoup d'applications telles que

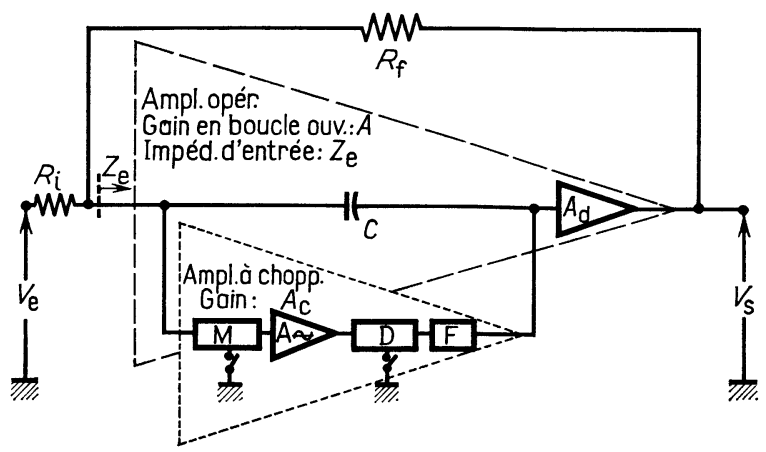

FIG. 4. - Stabilisation par choppers d'un amplificateur opérationnel.

$$
G=\frac{V_{\mathrm{g}}}{V_{\mathrm{e}}}=-\frac{R_{\mathrm{f}}}{R_{\mathrm{i}}} \frac{1}{1-\frac{1}{A}\left[1+\frac{R_{\mathrm{f}}}{Z_{\mathrm{e}}}+\frac{R_{\mathrm{f}}}{R_{\mathrm{i}}}\right]}
$$

$A_{\mathrm{d}}$, amplificateur continu de gain $A_{\mathrm{d}} ; \mathrm{A}_{\sim}$, amplificateur alternatif ; $M$, modulateur ; $D$, démodulateur $\mathrm{F}$, filtre.

servo-mécanismes ou stabilisation d'amplificateurs opérationnels. La figure 4 schématise cette dernière application à titre d'exemple : aux fréquences élevées, la capacité $C$ court-circuite l'amplificateur à choppers. Aux basses, ce dernier et l'amplificateur continu sont en cascade. Pour des gains de $A_{\mathrm{c}}=10^{3}$ et $A_{\mathrm{d}}=10^{4}$ par exemple, $A$ passe de $10^{7}$ aux basses à $10^{4}$ aux aiguës, ce qui, pour des relations de phase assez faciles à tenir avec ce système, assure la stabilisation (il faut, en particulier, que le module du gain en tension réinjectée à l'entrée tombe en dessous de 1 avant que le déphasage n'atteigne $\pi$ ).

Examinons d'abord le cas où l'amplificateur intermédiaire serait parfait : $G_{\mathbf{A}}(j \omega)=1$ quel que soit $\omega$. En posant encore :

$$
\lambda_{2}=\frac{1}{2}\left(\sqrt{\frac{\tau_{\mathrm{M} 2}}{\tau_{\mathrm{D} 2}}}+\sqrt{\frac{\tau_{\mathrm{D} 2}}{\tau_{\mathrm{M} 2}}}\right)
$$

il vient :

$$
\begin{aligned}
G_{\mathrm{p}}(j \omega)=\frac{(j \omega)^{2} \tau_{1}^{2}+2(j \omega) \lambda_{1} \tau_{1}+1}{\left(1+j \omega \tau_{\mathrm{M} 2}\right)\left(1+j \omega \tau_{\mathrm{D} 2}\right)} \\
=\frac{(j \omega)^{2} \tau_{1}^{2}+2(j \omega) \lambda_{1} \tau_{1}+1}{(j \omega)^{2} \tau_{2}^{2}+2(j \omega) \lambda_{2} \tau_{2}+1}=\frac{N}{D} .
\end{aligned}
$$

On remarque que le numérateur $N$ et le dénominateur $D$ ne sont autres que les inverses de fonctions de transfert de systèmes du second ordre, self $L$ et résistance $R$ à l'entrée en série et capacité $C$ en shunt à la sortie, où l'on a posé :

$\tau=\sqrt{L C}=1 / \omega_{\mathrm{r}} \quad\left(\omega_{\mathrm{r}}=\right.$ pulsation de résonance $)$

et

$\lambda=1 / 2 R / \sqrt{L / C} \quad(\lambda=$ facteur d'amortissement $)$.

Ces fonctions sont données sous forme réduite dans les manuels avec $\omega \tau$ comme variable et $\lambda$ comme 
paramètre. La figure 5 rappelle l'aspect du module en ordonnées logarithmiques et celui de la phase en coordonnées linéaires.

On remarque aussi que $\lambda_{2}$ est le rapport de la moyenne arithmétique à la moyenne géométrique

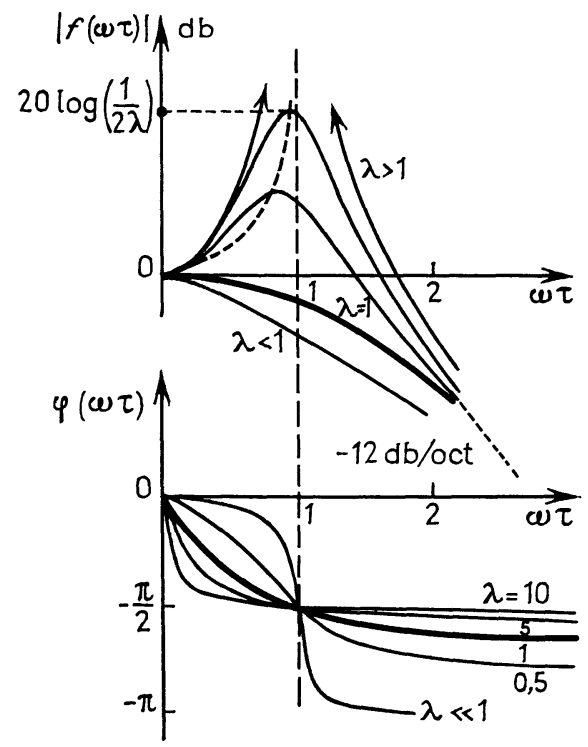

Fig. 5. - Fonction de transfert du système du second ordre.

Erratum : Sur le graphique du haut, lire : $\lambda<1$ en haut à droite, et $\lambda>1$ en bas à gauche.

de $\tau_{\mathrm{M} 2}$ et $\tau_{\mathrm{D} 2}$. Il est donc toujours supérieur ou égal à 1 et il en résulte que $\mathrm{D}$ est toujours apériodique, avec une courbe sans maximum. Par contre :

$$
\begin{aligned}
\lambda_{1}=\frac{1}{2}\left(\sqrt{\frac{\tau_{\mathrm{M} 1}}{\tau_{\mathrm{D} 1}}}+x \sqrt{\frac{\tau_{\mathrm{D} 1}}{\tau_{\mathrm{M} 1}}}\right) \\
=\sqrt{x} \frac{1}{2}\left(\sqrt{\frac{C_{1} \rho}{C_{2} R_{2}}}+\sqrt{\frac{C_{2} R_{2}}{C_{1} \rho}}\right)
\end{aligned}
$$

est inférieur à 1 sauf si $x \tau_{\mathrm{D} 1}$ diffère beaucoup de $\tau_{\mathrm{M} 1}$ car $x<1$.

La construction donnant $\left|G_{\mathrm{p}}\right|$ est alors la suivante (fig. 6).

Au voisinage de $\omega_{1}=1 / \tau_{1}$, abscisse du maximum de $1 /|N|$, la pente de $1 /|D|$ est encore faible, de telle sorte que l'abscisse du minimum de $\left|G_{\mathrm{p}}\right|$ diffère peu de $\omega_{1}$. En admettant $\omega_{1}$ comme valeur approchée de l'abscisse du minimum de $\left|G_{\mathrm{p}}\right|$, on ne commet sur ce minimum lui-même qu'une erreur très faible. On trouve ainsi :

$$
\begin{aligned}
\Delta A_{1 \mathrm{db}}=20 \log \left(2 \lambda_{1}\right)-10 \log & {\left[1+\left(\frac{\tau_{\mathrm{M} 2}}{\tau_{1}}\right)^{2}\right] } \\
& -10 \log \left[1+\left(\frac{\tau_{\mathrm{D} 2}}{\tau_{1}}\right)^{2}\right] .
\end{aligned}
$$

On a vu que quand $x$ décroît le gain en continu croît, mais l'expression de $\lambda_{1}$ montre qu'en même

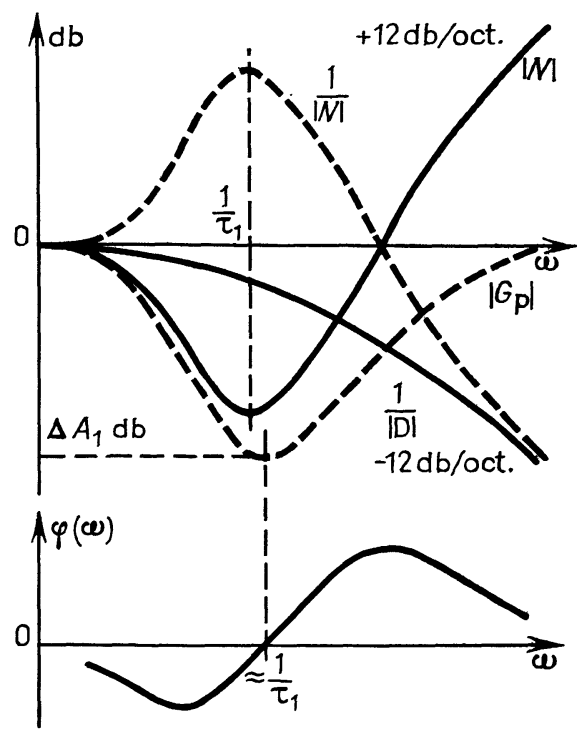

FIG. 6. - Calcul graphique du gain relatif.

temps $\lambda_{1}$ décroît, au détriment de la réponse en fréquence. Pour $x \geqslant 1 / 4, A_{1}$ peut couramment n'être que de 1 ou $2 \mathrm{db}$, mais pour $x \leqslant 1 / 10$ il peut dépasser $20 \mathrm{db}$. On recherchera le compromis nécessaire en calculant $\lambda_{1}$.

Après le passage au minimum, $\left|G_{\mathfrak{p}}\right|$ se rapproche de l'axe des $\omega$, restant dessous ou le coupant suivant que la quantité $\frac{\tau_{1}}{\sqrt{\tau_{\mathrm{M} 2} \tau_{\mathrm{D} 2}}}$ est inférieure ou supérieure
à 1 .

CAS DE L'AMPlificateur IMPARFait. - Considérons maintenant le cas plus proche de la réalité où $G_{\mathbf{A}}(j \omega)$ serait un produit de facteurs de la forme $j \omega \tau_{\mathbf{i}} /\left(1+j \omega \tau_{\mathbf{i}}\right)$

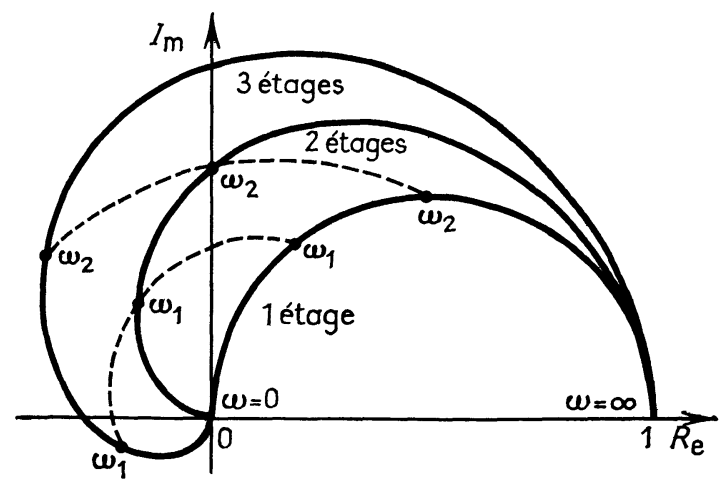

FIG. 7. - Gain relatif complexe d'une chaîne à plusieurs étages $R-C$.

représentant le gain relatif complexe d'une chaîne d'étages couplés par résistance-capacité, gain dont la figure 7 donne l'allure dans le plan complexe pour 1, 2 


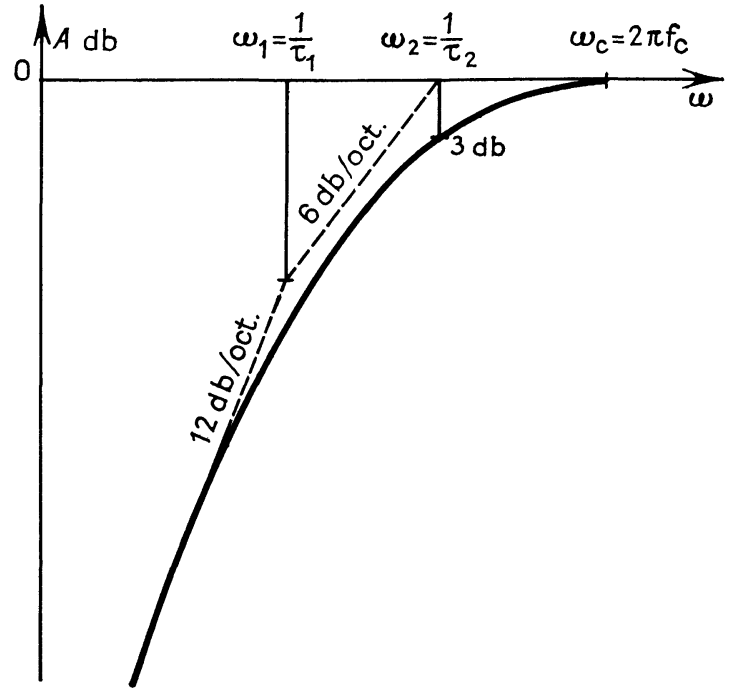

FIG. 8. - Module du gain relatif dans le cas de deux étages.

et 3 facteurs, et dont la figure 8 donne l'allure du module dans le cas de deux facteurs. Le numérateur complet de $G_{\mathrm{p}}$ peut s'écrire :

$$
\begin{aligned}
& N=\underbrace{(j \omega)^{2} \tau_{1}^{2}}_{V_{\mathbf{B}}} \underbrace{G_{\mathbf{A}}(j \omega)}_{V_{\mathbf{A}}}-\underbrace{x(j \omega)^{2} \tau_{1} G_{\mathbf{A}}(j \omega)}_{-x V_{\mathbf{B}}} \underbrace{}_{V_{\mathbf{A}}} \\
& +\underbrace{x(j \omega)^{2} \tau_{1}^{2}}_{x V_{\mathbf{B}}}+\underbrace{2 j \omega \lambda_{1} \tau_{1}}_{V_{\mathbf{O}}}+1 \text {. }
\end{aligned}
$$

$V_{\mathrm{A}}, V_{\mathrm{B}}$ et $V_{\mathrm{C}}$ sont des vecteurs du plan complexe, les accolades indiquant la correspondance des notations. $V_{\mathrm{B}}$ est réel négatif et proportionnel à $\omega^{2}, V_{\mathrm{C}}$ est imaginaire pur vers le haut, proportionnel à $\omega$, et $V_{\mathrm{A}}$ complexe suit une loi du type donné figure 7 . Une courbe de ce type étant choisie, il est facile de construire $N$. On a d'abord, figure 9, tracé $N$ dans le cas simplifié où $G_{\mathrm{A}}$ est identiquement égal à 1 . Le lieu de $N$ est la parabole $x=y^{2} / 2 \lambda_{1}^{2}$. Les traits pleins correspondent à $\omega=1 / \tau_{1}$, valeur pour laquelle $N$ se réduit à $N_{1}=2 j \lambda_{1}$. Les flèches pointillées montrent le sens de variation pour $\omega$ croissant.

On a ensuite, figure 10 , construit $N$ en prenant pour $G_{\mathrm{A}}$ une loi du type de la figure 7 . Les vecteurs y figurent dans la position $\omega=1 / \tau_{1}$, comme sur la figure 9. On voit en pointillé le chemin de sommation : $0+V_{\mathrm{B}} V_{\mathrm{A}},-x V_{\mathrm{B}} V_{\mathrm{A}},+x V_{\mathrm{B}},+V_{\mathrm{C}},+1$, qui abou- tit à $N_{1}$. Or, si les vecteurs $+1, x V_{\mathrm{B}}$ et $V_{\mathrm{C}}$ sont inchangés, le vecteur $V_{\mathrm{B}}$ est remplacé par $V_{\mathrm{B}} V_{\mathrm{A}}$ et le vecteur $-x V_{\mathrm{B}}$ par $-x V_{\mathrm{B}} V_{\mathrm{A}}$. Appelons $P$ le vecteur $V_{\mathrm{B}} V_{\mathrm{A}}-V_{\mathrm{B}}$ et $Q$ le vecteur $\left(-x V_{\mathrm{B}} V_{\mathrm{A}}\right)-\left(-x V_{\mathrm{B}}\right)$. Il est bien évident que $N_{1}$, au lieu de coïncider avec $V_{\mathrm{C}}$ comme sur la figure 9 , s'en déduit par addition de $P+Q$. Or $Q=-x P$, donc le vecteur à ajouter à $V_{\mathrm{C}}$ pour obtenir $N_{1}$ n'est autre que $P(1-x)$ que l'on voit à gauche de la figure.
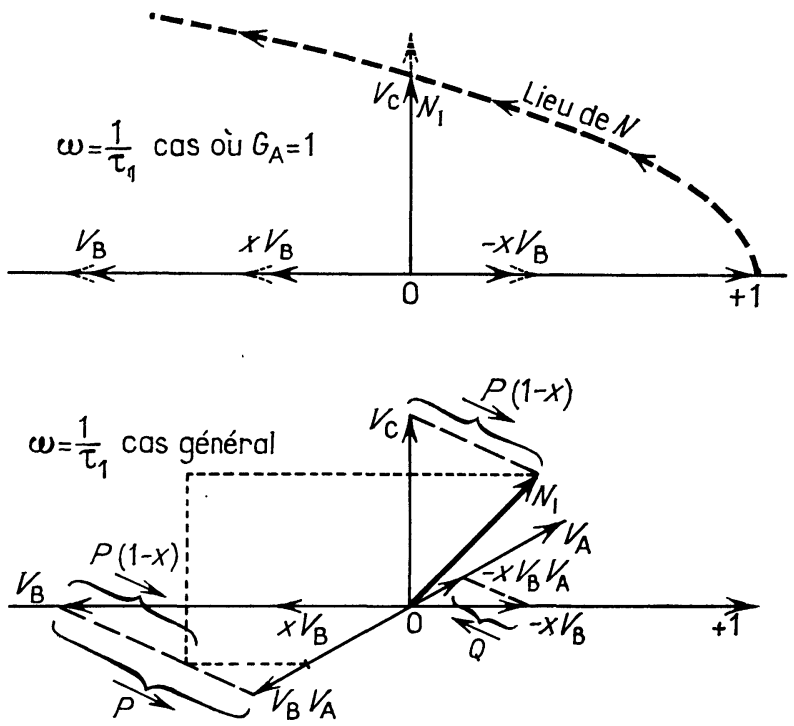

Fig. 9 et 10 . - Variation du déphasage due à l'imperfection de l'amplificateur.

Ceci montre que, pour $\omega=\omega_{1}, N$ est en retard sur $V_{\mathrm{C}}$, avec une amplitude du même ordre, alors que ces deux vecteurs coïncidaient quand on admettait $G_{\mathrm{A}}=1$. Les conclusions résumées par la figure 6 sont alors modifiées en ce sens que, dans le cas où $G_{\mathrm{A}} \neq 1$, le déphasage de $G_{\mathrm{p}}$ s'annule non plus à l'abscisse du minimum mais à une abscisse supérieure $\left(^{\mathbf{1}}\right)$.

(1) Les figures 1 et 4 et les formules exprimant le gain sont extraites de Electrical Design News Magazine, mars 1956, p. 98-101.

Manuscrit reçu le 22 mai 1967. 